\title{
An antagonist of growth hormone-releasing hormone protects against LPS-induced increase of bronchoalveolar lavage fluid protein concentration
}

\author{
Mohammad S. Akhter ${ }^{1} \cdot$ Khadeja-Tul Kubra $^{1} \cdot$ Mohammad A. Uddin $^{1} \cdot$ Seetharama Jois $^{1} \cdot$ Nektarios Barabutis $^{1}$ (I)
}

Received: 8 November 2021 / Revised: 28 November 2021 / Accepted: 30 November 2021 / Published online: 7 January 2022

(c) The Author(s), under exclusive licence to Springer Nature Switzerland AG 2021

\begin{abstract}
Growth Hormone-Releasing Hormone (GHRH) is a neuropeptide regulating the release of Growth Hormone (GH) from the anterior pituitary gland, and acts as a growth factor in a diverse variety of tissues. GHRH antagonists (GHRHAnt) have been developed to counteract those events, and the beneficial effects of those peptides toward homeostasis have been associated with anti-inflammatory activities. Our lab is interested in delineating the mechanisms governing endothelial barrier function. Our goal is to establish new grounds on the development of efficient countermeasures against Acute Respiratory Distress Syndrome (ARDS), which has been associated with thousands of deaths worldwide due to COVID-19. Herein we demonstrate in vivo that GHRHAnt suppresses LPS-induced increase in bronchoalveolar lavage fluid (BALF) protein concentration, thus protecting the lungs against edema and inflammation.
\end{abstract}

Keywords Inflammation $\cdot$ Lung $\cdot$ Permeability

\section{Introduction}

GHRH is consisted of 44 amino acids [1] and exerts extrahypothalamic effects in peripheral tissues including brain, heart, and retina. Receptors specific for that hormonepituitary-type GHRH receptor (GHRH-R) and its splice variants have been detected in various tissues including the lungs, supporting the multifaceted role of GHRH in human physiology [2].

GHRH is associated with inflammatory responses. In human ciliary epithelial cells, NF- $\mathrm{kB}$ subunit p65 is phosphorylated by lipopolysaccharides (LPS), resulting in GHRH-R upregulation. GHRH triggers IL-6 release from human peripheral blood mononuclear cells, and enhances immune cell function in the elderly. Moreover, cytokine production in ciliary and iris epithelial cells is mediated by GHRH-R during LPS-induced occular inflammation [3].

Responsible Editor: John Di Battista.

Nektarios Barabutis

barabutis@ulm.edu

1 School of Basic Pharmaceutical and Toxicological Sciences, College of Pharmacy, University of Louisiana Monroe, 1800 Bienville Drive, Monroe, LA 71201, USA
The endothelial barrier regulates the exchange of blood fluid, electrolytes and proteins across the vascular wall. This structure is affected by several stimuli, including LPS, growth factors, and SARS-CoV-2 [4]. Those factors affect several intracellular messengers (kinases, phosphatases) to induce cytoskeletal remodeling by modulating junction and adhesion proteins. Those changes affect endothelial barrier function to increase lung permeability across the endothelial and epithelial barriers [4-6].

Vascular endothelial dysfunction is a central event in the pathogenesis of sepsis and ARDS. Lung hyper-permeability allows fluid, proteins, and macromolecules into the alveolar airspace, resulting in increased BALF protein concentration [7, 8]. GHRHAnt enhance endothelial barrier function in vitro $[9,10]$, but their effects in an in vivo model of murine lung injury in the context of BALF protein concentration have not been investigated yet.

\section{Materials and methods}

\section{Reagents}

GHRHAnt (\#4030691) was purchased from Bachem (Torrance, CA) and LPS from Sigma-Aldrich (St Louis, MO). 
The scramble peptide was synthesized in LSU AG Center Biotechnology Lab, Baton Rouge, LA (Invoice No.:1075).

\section{Animals}

Seven-week-old C57BL/6 (male) mice were purchased from Envigo (Indianapolis, IN) and maintained in 12:12-h light/dark cycle. The temperature $\left(22-24{ }^{\circ} \mathrm{C}\right)$ and humidity $(50-60 \%)$ were in control, and all procedures were approved by the Institutional Animal Care and Use Committee (IACUC).

\section{Experimental procedures}

Mice were treated with intratracheal (I.T.) injection of saline or LPS $(1.6 \mathrm{mg} / \mathrm{kg}$ ) and were post-treated (24 h after LPS) with intravenous (I.V.) (tail vein) injection of either vehicle; or scramble peptide (100 $\mu \mathrm{g} / \mathrm{kg})$; or GHRHAnt $(100 \mu \mathrm{g} / \mathrm{kg})$. Protein concentration was measured by the bicinchoninic acid protein assay kit.

\section{Statistics}

The data are expressed as mean values \pm SEM (standard error of mean). Student's $t$ test was used to determine statistically significant differences among groups and GraphPad Prism (version 5.01) to analyze the data.

\section{Results and discussion}

Figure 1 suggests that LPS increases BALF protein concentration of LPS-treated mice as compared to the control group. GHRHAnt significantly reduces BALF protein concentration in LPS-pretreated mice, while the scramble peptide for GHRHAnt did not exert any significant effects. Hence, we suggest a protective role of GHRHAnt against LPS-induced injury.

GHRH-R regulates key signaling pathways involved in lung growth and inflammation. GHRHAnt inhibit lung inflammation and fibrosis in bleomycin-induced fibrosis model in vivo and suppress several inflammatory pathways (i.e., ERK, JAK2/STAT3, NF-кB) [9, 11]. They also suppress cofilin and myosin light chain 2 (MLC2) activation to regulate cytoskeletal remodeling in LPS-induced inflammation [12]. Those events increase the transendothelial resistance of endothelial monolayers to enhance barrier integrity [9].

Inflammatory conditions are characterized by excessive generation of reactive oxygen species (ROS) which cause disruption of inter-endothelial junctions, endothelial hyperpermeability and abnormal extravasation of proteinous fluids and macromolecules to the lungs [13]. Those events cause

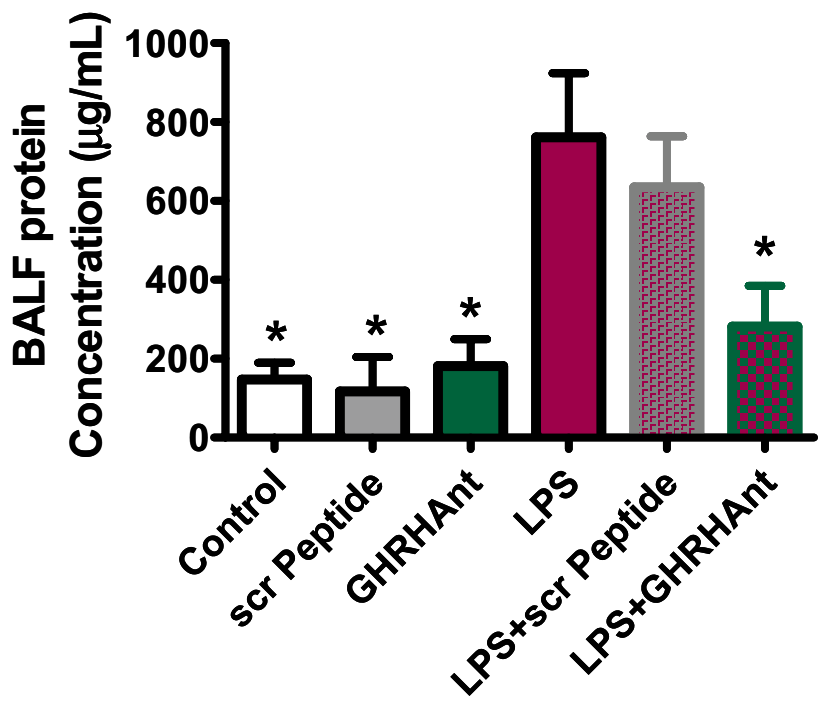

Fig. 1 GHRHAnt reduces BALF protein concentration of mice exposed to LPS. Male C57BL/6 mice were treated with an intratracheal (I.T.) injection of saline or LPS (1.6 mg/kg, dissolved in saline), and were post-treated ( $24 \mathrm{~h}$ after LPS) with an intravenous (I.V.) (tail vein) injection of either vehicle (0.1\% DMSO in 10\% 1,2-propanediol); or scramble peptide ( $100 \mu \mathrm{g} / \mathrm{kg}$, dissolved in $0.1 \%$ DMSO and 10\% 1,2-propanediol); or GHRHAnt $(100 \mu \mathrm{g} / \mathrm{kg}$, dissolved in $0.1 \%$ DMSO and 10\% 1,2-propanediol). $48 \mathrm{~h}$ after LPS, BALF from the lungs was obtained by instilling and withdrawing $1 \mathrm{ml}$ of PBS using tracheal cannula. ${ }^{*} P<0.05$ vs LPS, $n=3$ animals per group. Means \pm SEM

edema formation and infiltration of inflammatory cells. GHRHAnt exert anti-oxidative effects in lung and brain endothelial cells and inhibit lipid and protein oxidation [2]. In contrast, GHRH increases the expression of cyclooxygenase-2, cytochrome $c$ oxidase-IV and inducible nitric oxide synthase, which are involved in ROS generation [2].

Unfolded protein response (UPR) has been associated with endothelial barrier function. Mild UPR activation protects the endothelial barrier, while Kifunensin (UPR suppressor) induces endothelial permeability [12]. P53 mediates, at least in part, those UPR-mediated activities. Indeed, the lungs of P53 knockout mice are more vulnerable to LPS as compared to the wild-type counterparts, suggesting a crucial role of P53 against pulmonary inflammation [2]. P53 can also suppress the lipid peroxidation marker malondialdehyde (MDA) by reducing endothelial ROS generation $[14,15]$. Since GHRHAnt activate UPR [10] and induce P53 expression in those cells [9], the interrelation of GHRH, UPR and P53 may regulate the microvasculature. Further studies employing genetically modified mice which express more or less of UPR will most probably deliver new possibilities to oppose endothelial barrier dysfunction. 
Funding We are supported by (1) R\&D, Research Competitiveness Subprogram (RCS) of Louisiana Board of Regents through Board of Regents Support Fund (LEQSF(2019-22)-RD-A-26) (P.I: NB) (2) Faculty Research Support Program from College of Pharmacy, ULM (P.I: NB) (3) National Institute of General Medical Sciences of the National Institutes of Health (5P20 GM103424-20).

Data availability statement The datasets are available from the corresponding author on reasonable request.

\section{Declarations}

Conflict of interest None.

\section{References}

1. Schally AV, et al. Actions and potential therapeutic applications of growth hormone-releasing hormone agonists. Endocrinology. 2019;160(7):1600-12.

2. Akhter MS, Barabutis N. Suppression of reactive oxygen species in endothelial cells by an antagonist of growth hormone-releasing hormone. J Biochem Mol Toxicol. 2021;35(10): e22879.

3. Ren JL, et al. Growth hormone-releasing hormone receptor mediates cytokine production in ciliary and iris epithelial cells during LPS-induced ocular inflammation. Exp Eye Res. 2019;181:277-84.

4. Barabutis N, Verin A, Catravas JD. Regulation of pulmonary endothelial barrier function by kinases. Am J Physiol Lung Cell Mol Physiol. 2016;311(5):L832-45.

5. Sukriti S, et al. Mechanisms regulating endothelial permeability. Pulm Circ. 2014;4(4):535-51.
6. Komarova YA, et al. Protein interactions at endothelial junctions and signaling mechanisms regulating endothelial permeability. Circ Res. 2017;120(1):179-206.

7. Claesson-Welsh L, Dejana E, McDonald DM. Permeability of the endothelial barrier: identifying and reconciling controversies. Trends Mol Med. 2021;27(4):314-31.

8. Duong CN, Vestweber D. Mechanisms ensuring endothelial junction integrity beyond VE-cadherin. Front Physiol. 2020;11:519.

9. Uddin MA, et al. GHRH antagonists support lung endothelial barrier function. Tissue Barriers. 2019;7(4):1669989.

10. Akhter MS, et al. Involvement of the unfolded protein response in the protective effects of growth hormone releasing hormone antagonists in the lungs. J Cell Commun Signal. 2021;15(1):125-9.

11. Zhang $\mathrm{C}$, et al. Growth hormone-releasing hormone receptor antagonist modulates lung inflammation and fibrosis due to bleomycin. Lung. 2019;197(5):541-9.

12. Barabutis N. Growth hormone releasing hormone in endothelial barrier function. Trends Endocrinol Metab. 2021;32(6):338-40.

13. He P, Talukder MAH, Gao F. Oxidative stress and microvessel barrier dysfunction. Front Physiol. 2020;11:472.

14. Akhter MS, Uddin MA, Barabutis N. P53 Regulates the redox status of lung endothelial cells. Inflammation. 2020;43(2):686-91.

15. Akhter MS, et al. P53-induced reduction of lipid peroxidation supports brain microvascular endothelium integrity. J Pharmacol Sci. 2019;141(1):83-5.

Publisher's Note Springer Nature remains neutral with regard to jurisdictional claims in published maps and institutional affiliations. 